EPJ Web of Conferences 71, 00008 (2014)

DOI: $10.1051 /$ epjconf / 20147100008

(C) Owned by the authors, published by EDP Sciences, 2014

\title{
Kaons at CERN: recent results and prospects
}

\author{
Giuseppina Anzivino1,a \\ ${ }^{1}$ Department of Physics, University of Perugia and INFN
}

\begin{abstract}
The NA48/2 and NA62 experiments at CERN study rare kaon decays using the decay in flight technique. In this paper two selected topics will be presented: new measurements performed by the two experiments of the $K^{ \pm} \rightarrow \pi^{ \pm} \gamma \gamma$ decay which represents a stringent test of the Chiral Perturbation Theory and the measurement of the Branching Ratio of the very rare decay $K^{+} \rightarrow \pi^{+} v \bar{v}$ at $10 \%$ level, that will provide a test of the Standard Model and possible extensions beyond it.
\end{abstract}

\section{Introduction}

The study of kaons has always been an important source for the investigation of the flavour sector of fundamental interactions and for the understanding of the symmetries of nature.

Since the discovery of CP violation in 1964 in $K^{0} \rightarrow \pi^{+} \pi^{-}[1]$ kaons remain a privileged observatory for flavour physics phenomena where all the features of flavour physics are present: kaons are a rather simple system of the lightest mesons containing a quark flavour of a generation beyond the first, they have rather long lifetimes and are produced in large quantity. In the era of the high energy frontier, the flavour sector can probe the high energy scale exploiting the precision frontier, complementary to the high energy one. In addition, processes that are suppressed in the Standard Model (SM) are very sensitive to New Physics (NP), therefore the study of rare kaon decays can provide a deeper insight in fundamental physics.

\footnotetext{
${ }^{1}$ on behalf of the NA62 and NA48/2 Collaborations: F. Ambrosino, A. Antonelli, G. Anzivino, R. Arcidiacono, W. Baldini, S. Balev, J.R. Batley, M. Behler, S. Bifani, C. Biino, A. Bizzeti, B. Bloch-Devaux, G. Bocquet, V. Bolotov, F. Bucci, N. Cabibbo, M. Calvetti, N. Cartiglia, A. Ceccucci, P. Cenci, C. Cerri, C. Cheshkov, J.B. Chèze, M. Clemencic, G. Collazuol, F. Costantini, A. Cotta Ramusino, D. Coward, D. Cundy, A. Dabrowski, G. D’Agostini, P. Dalpiaz, C. Damiani, H. Danielsson, M. De Beer, G. Dellacasa, J. Derré, H. Dibon, D. Di Filippo, L. DiLella, N. Doble, V. Duk, J. Engelfried, K. Eppard, V. Falaleev, R. Fantechi, M. Fidecaro, L. Fiorini, M. Fiorini, T. Fonseca Martin, P.L. Frabetti, A. Fucci, S. Gallorini, L. Gatignon, E. Gersabeck, A. Gianoli, S. Giudici, A. Gonidec, E. Goudzovski, S. Goy Lopez, E. Gushchin, B. Hallgren, M. Hita-Hochgesand, M. Holder, P. Hristov, E. Iacopini, E. Imbergamo, M. Jeitler, G. Kalmus, V. Kekelidze, K. Kleinknecht, V. Kozhuharov, W. Kubischta, V. Kurshetsov, G. Lamanna, C. Lazzeroni, M. Lenti, E. Leonardi, L. Litov, D. Madigozhin, A. Maier, I. Mannelli, F. Marchetto, G. Marel, M. Markytan, P. Marouelli, M. Martini, L. Masetti, P. Massarotti, E. Mazzucato, A. Michetti, I. Mikulec, M. Misheva, N. Molokanova, E. Monnier, U. Moosbrugger, C. Morales Morales, M. Moulson, S. Movchan, D.J. Munday, M. Napolitano, A. Nappi, G. Neuhofer, A. Norton, T. Numao, V. Obraztsov, V. Palladino, M. Patel, M. Pepe, A. Peters, F. Petrucci, M.C. Petrucci, B. Peyaud, R. Piandani, M. Piccini, G. Pierazzini, I. Polenkevich, I. Popov, Yu. Potrebenikov, M. Raggi, B. Renk, F. Retière, P. Riedler, A. Romano, P. Rubin, G. Ruggiero, A. Salamon, G. Saracino, M. Savrié, M. Scarpa, V. Semenov, A. Sergi, M. Serra, M. Shieh, S. Shkarovskiy, M.W. Slater, M. Sozzi, T. Spadaro, S. Stoynev, E. Swallow, M. Szleper, M. Valdata-Nappi, P. Valente, B. Vallage, M. Velasco, M. Veltri, S. Venditti, M. Wache, H. Wahl, A. Walker, R. Wanke, L. Widhalm, A. Winhart, R. Winston, M.D. Wood, S.A. Wotton, O. Yushchenko, A. Zinchenko, M. Ziolkowski.
}

ae-mail: giuseppina.anzivino@pg.infn.it

This is an Open Access article distributed under the terms of the Creative Commons Attribution License 2.0, which permits unrestricted use, distribution, and reproduction in any medium, provided the original work is properly cited. 


\section{The $K^{ \pm} \rightarrow \pi^{ \pm} \gamma \gamma$ decay}

The Chiral Perturbation Theory (ChPT) provides a systematic approach to the intrinsically nonperturbative nature of the strong interactions in kaon physics. Radiative non-leptonic kaon decays provide a crucial test of this theory and can give information on the structure of hadronic interactions at low energy. The contributions of the chiral lagrangian to this decay [2] appear at $O\left(p^{4}\right)$, where only the $\Delta I=1 / 2$ invariant amplitudes $A(z)$ and $C(z)$, with $z=m_{\gamma \gamma}^{2} / m_{K^{ \pm}}^{2}$, contribute. The decay rate and the spectrum strongly depend on a single parameter $\hat{c}$ predicted to be $O(1)$. The invariant $m_{\gamma \gamma}$ distribution is favored above $2 m_{\pi^{+}}$and exibits a cusp at $2 m_{\pi^{+}}$threshold. Calculations at $O\left(p^{6}\right)$ [3] show that unitarity correction modify the decay spectrum significantly, leading, in particular, to non-zero differential decay rate at zero di-photon invariant mass .

The ChPT predictions for the decay spectra for values of $\hat{c}=-2 ; 0 ; 2$ are shown in Fig. 1 for $O\left(p^{4}\right)$ and $O\left(p^{6}\right)$ parametrization: the diphoton mass spectra exhibit a characteristic cusp at twice the pion mass due to the dominant pion loop amplitude.
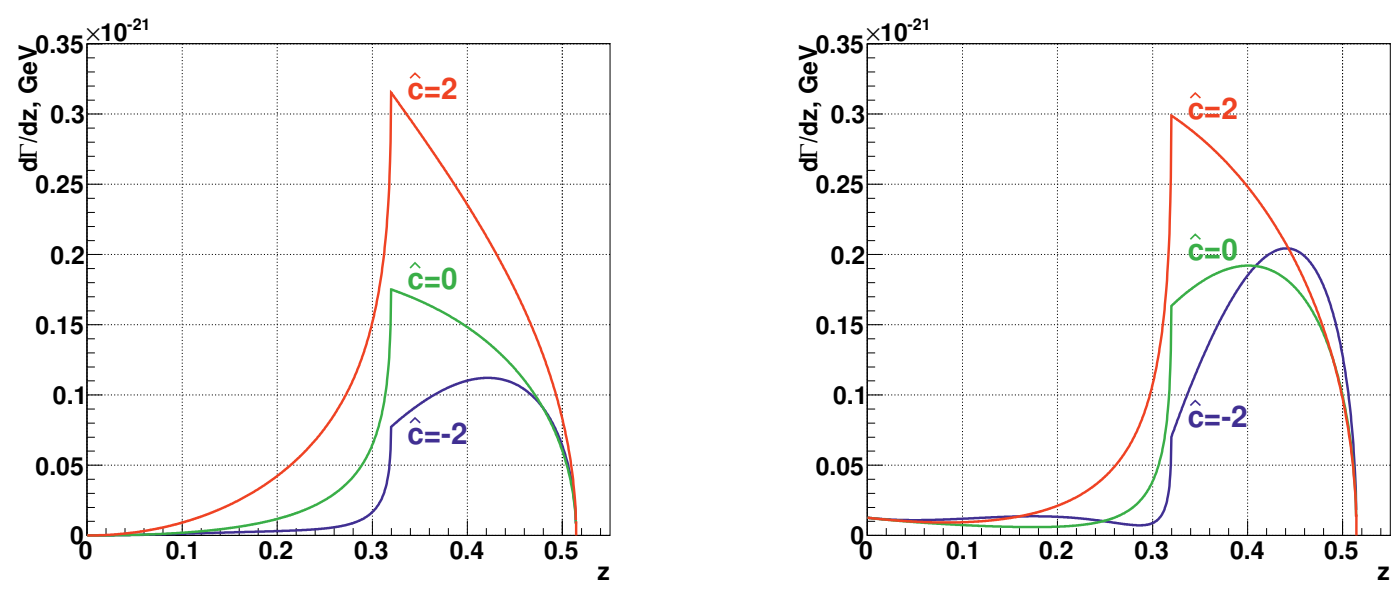

Figure 1. ChPT predictions for $K^{ \pm} \rightarrow \pi^{ \pm} \gamma \gamma$ differential rate in terms of $z=m_{\gamma \gamma}^{2} / m_{K^{ \pm}}^{2}$ for $O\left(p^{4}\right)$ (left) and $O\left(p^{6}\right)$ (right) parametrization [3].

On the experimental side, there is only one published result by the BNL E787 experiment [4], based on $31 K^{ \pm} \rightarrow \pi^{ \pm} \gamma \gamma$ candidates, in the kinematic region $100 \mathrm{MeV} / \mathrm{c}<p_{\pi}^{*}<180 \mathrm{MeV} / \mathrm{c}\left(p_{\pi}^{*}\right.$ is the $\pi^{+}$momentum in the $K^{+}$frame).

\subsection{Beam, detector and data taking}

The NA48/2 and NA62 experiments used the same beam line and detector. However beam line parameters and spectrometer magnet transverse momentum kick were significantly different. Simultaneous $K^{+}$and $K^{-}$beams were produced by $400 \mathrm{GeV} / \mathrm{c}$ primary SPS protons impinging on a beryllium target. Charged particles were selected by an achromatic system of four dipole magnets, which split the two beams in the vertical plane and recombined them on a common axis. The resulting beam momenta were of $(60 \pm 3) \mathrm{GeV} / \mathrm{c}$ for NA48/2 and $(74 \pm 1.4)$ for NA62. The momenta of $K^{ \pm}$decay products were measured by a magnetic spectrometer, housed in a tank filled with helium at nearly atmospheric pressure, located downstream of the decay vacuum tank. The spectrometer consisted of four drift 
chambers $(\mathrm{DCH})$ with a transverse width of $2.9 \mathrm{~m}$ : DCH1, DCH2 located upstream and DCH3, DCH4 downstream of a dipole magnet that provided a horizontal transverse momentum kick of 120 $\mathrm{MeV} / \mathrm{c}(265 \mathrm{MeV} / \mathrm{c})$ and a momentum resolution for the decay products of $\sigma_{p} / p=(1.02 \oplus 0.044 \cdot p) \%$ $\left(\sigma_{p} / p=(0.048 \oplus 0.009 \cdot p) \%\right)$, where $p$ is expressed in $\mathrm{GeV}$, for NA48/2 (NA62).

A hodoscope (HOD) consisting of two planes of orthogonal plastic scintillator strips, with each plane arranged in four quadrants, was placed after the spectrometer. The HOD provided trigger signals and time measurements of charged particles with a resolution of about 150 ps. Further downstream a liquid krypton electromagnetic calorimeter ( $\mathrm{LKr}$ ) with an active volume of $7 \mathrm{~m}^{3}$ of liquid krypton, $27 X_{0}$ deep, segmented transversally into 13248 projective $2 \times 2 \mathrm{~cm}^{2}$ cells and with no longitudinal segmentation. The LKr energy resolution was $\left.\left(\sigma_{E} / E=(3.2 / \sqrt{(} E) \oplus 9 / E \oplus 0.42\right) \%\right)$ and its spatial resolution for the transverse coordinates $x$ and $y$ of an isolated electromagnetic shower was $\left(\sigma_{x}=\right.$ $\left.\sigma_{y}=(4.2 / \sqrt{(} E) \oplus 0.6\right) \mathrm{mm}$.

A detailed description of the detector can be found in [5].

The preliminary results presented in this paper are based on data collected in two different runs: a 3-day NA48/2 special run in 2004 and a 3-month NA62 run in 2007. The two data sets are comparable in size, since a downscaling factor of $\sim 20$ on the trigger was active in 2007. Events have been selected in the region $z=m_{\gamma \gamma}^{2} / m_{K^{ \pm}}^{2}>0.2$ in order to minimize the $K^{ \pm} \rightarrow \pi^{ \pm} \pi^{0}$ background. We observed 149 (175) decay candidates in the 2004 (2007) data set with a 10\% (7\%) background contamination.

Fig. 2 shows the $K^{ \pm} \rightarrow \pi^{ \pm} \gamma \gamma$ spectra for the invariant mass and the $z$ variable (data and MC expectations) for signal and background for 2004 data set; the cusp at $2 m_{\pi^{+}}$threshold is evident, as expected by the ChPT predictions. The 2007 data set shows similar results.
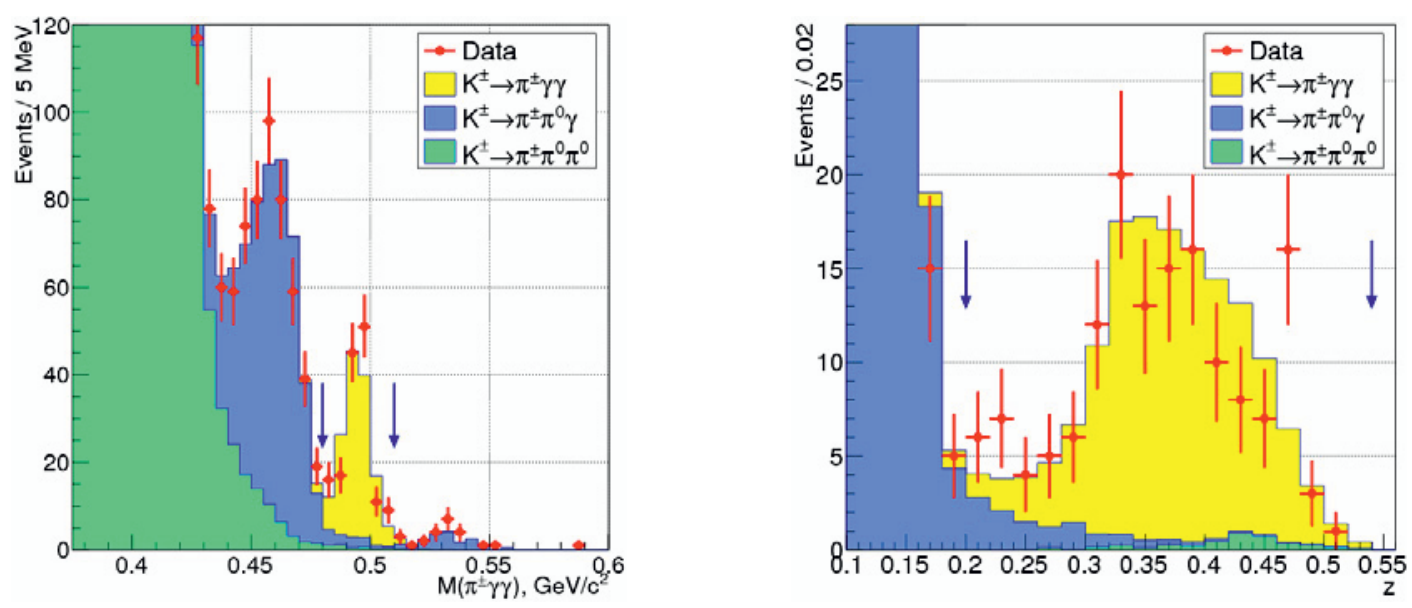

Figure 2. Results for the 2004 data set. Left: the $K^{ \pm} \rightarrow \pi^{ \pm} \gamma \gamma$ invariant mass spectrum with MC expectations for signal and background. Right: the $z=m_{\gamma \gamma}^{2} / m_{K^{ \pm}}^{2}$ spectrum with MC expectations for signal and background. Arrows indicate the signal region.

The preliminary 2004 and 2007 results and the combined result, that takes into account the correlation of the systematic uncertainties between the two data sets, are reported in Table 1.

The results are in agreement with the BNL E787 ones; the $O\left(p^{4}\right)$ and $O\left(p^{6}\right)$ models cannot be discriminated within the current experimental sensitivity. 
Table 1. Preliminary results of the fits to the ChPT parametrization. The model dependent BR values are computed on the full kinematic range.

\begin{tabular}{lccc}
\hline & 2004 data & 2007 data & combined \\
\hline$\hat{c}, O\left(p^{4}\right)$ fit & $1.36 \pm 0.33_{\text {stat }} \pm 0.07_{\text {syst }}$ & $1.71 \pm 0.29_{\text {stat }} \pm 0.06_{\text {syst }}$ & $1.56 \pm 0.22_{\text {stat }} \pm 0.07_{\text {syst }}$ \\
$\hat{c}, O\left(p^{6}\right)$ fit & $1.67 \pm 0.39_{\text {stat }} \pm 0.09_{\text {syst }}$ & $2.21 \pm 0.31_{\text {stat }} \pm 0.08_{\text {syst }}$ & $2.00 \pm 0.24_{\text {stat }} \pm 0.09_{\text {syst }}$ \\
$\mathrm{BR}, O\left(p^{6}\right)$ fit & $(0.94 \pm 0.08) \times 10^{-6}$ & $(1.06 \pm 0.07) \times 10^{-6}$ & $(1.01 \pm 0.06) \times 10^{-6}$ \\
\hline
\end{tabular}

A model-independent measurement of the $z$ spectrum in the kinematic range $z>0.2$ has been performed for the NA48/2 data set. The partial branching fractions in bins of the $z$ variable have been measured and a model-independent $B R$ has been computed by summing over the $z$ bins:

$$
B R_{M I}(z>0.2)=\left(0.877 \pm 0.087_{\text {stat }} \pm 0.017_{\text {syst }}\right) \times 10^{-6}
$$

\section{The $K^{+} \rightarrow \pi^{+} v \bar{v}$ decay}

The rare decays $K^{+} \rightarrow \pi^{+} v \bar{v}$ and $K_{L} \rightarrow \pi^{0} v \bar{v}$ are extremely attractive processes to study the physics of flavour mixing since both are exceptionally clean modes. These decays are strongly suppressed in the SM, dominated by short-distance dynamics and top quark loop contributions. Moreover, the shortdistance amplitude is governed by one single semileptonic operator whose hadronic matrix element can be determined experimentally using the semileptonic kaon decay; therefore the main hadronic uncertainties can be eliminated by experimental data. The two "golden" rare kaon modes offer unique opportunities for testing the Standard Model and deepening our knowledge of the CKM matrix, complementary to those in B decays. Furthermore, they are extremely sensitive to possible new degrees of freedom beyond the Standard Model. At the quark level the two processes arise from the $s \rightarrow d v \bar{v}$ process, which in the SM originates from a double $W^{ \pm}$exchange (the first graph in Fig. 3) and a combination of $Z^{0}$ penguins (the second and third graph in Fig. 3).
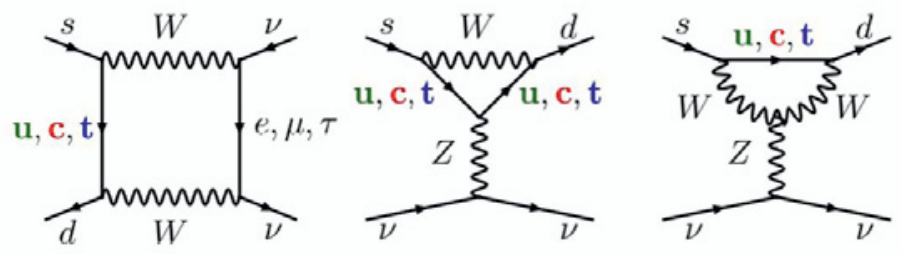

Figure 3. Graphs for $s \rightarrow d v \bar{v}$ in the Standard Model

The SM prediction [6] for the $K^{+} \rightarrow \pi^{+} v \bar{v}$ channel is $B R=\left(7.81_{-0.71}^{+0.80} \pm 0.29\right) \times 10^{-11}$. The error comes mainly from the uncertainty in the CKM matrix elements, while the irreducible theoretical uncertainty amounts to less than $2 \%$. The extreme theoretical cleanness of these decays remains also in new physics scenarios like Minimal Flavour Violation (MFV)[7] or non-MFV models[8] and even not large deviations from the SM value (for example around 20\%) can be considered signals of new physics.

From the experimental side, the decay $K^{+} \rightarrow \pi^{+} v \bar{v}$ has been observed by the experiments E787 and E949 at the BNL, using a stopping kaon technique. The combined result from the two experiments 
for branching ratio is $B R=1.73_{1.05}^{1.15} \times 10^{-10}$ [9]. The measured central value is about twice as large as, but still compatible with the SM expectations within the errors. It is clear that only a measurement of the branching ratio with at least $10 \%$ accuracy can be a significant test of the validity of the SM and of the possible existence of new physics. This is the main goal of the NA62 experiment at CERNSPS[10]. It aims to collect order of $100 \mathrm{~K}^{+} \rightarrow \pi^{+} v \bar{v}$ events in about two years of data taking, keeping a background contamination around $10 \%$.

\subsection{Experimental strategy}

The measurement of the $K^{+} \rightarrow \pi^{+} v \bar{v}$ Branching Ratio is very challenging due to the weak experimental signature (only one charged track in the final state) and huge background.

The two undetectable neutrinos in the final state require the design of an experiment with redundant measurement of the event kinematics and hermetic vetoes to achieve a background rejection $S / B \simeq 10$. Particular care has to be taken to suppress the two-body decays $K^{+} \rightarrow \pi^{+} \pi^{0}$ and $K^{+} \rightarrow \mu^{+} v$ which have branching ratios up to $10^{10}$ times larger than the expected signal and can mimic the signal signature if a muon is misidentified as a pion or in case of loss of the photons from the $\pi^{0}$ decay. Backgrounds from $K^{+}$three- and four-body decays are also potentially dangerous. The most frequent $K^{+}$decay modes are listed in Table 2 .

Table 2. The most frequent $K^{+}$decay channels.

$\begin{array}{lc}\text { Decay mode } & \text { Branching Ratio } \\ K^{+} \rightarrow \mu^{+} v & 63.5 \% \\ K^{+} \rightarrow \pi^{+} \pi^{0} & 20.7 \% \\ K^{ \pm} \rightarrow \pi^{ \pm} \pi^{+} \pi^{-} & 5.6 \% \\ K^{+} \rightarrow \pi^{0} e v & 5.1 \% \\ K^{+} \rightarrow \pi^{0} \mu v & 3.3 \% \\ K^{ \pm} \rightarrow \pi^{+} \pi^{-} \pi^{0} & 1.8 \%\end{array}$

In order to achieve the design goal, high statistic is needed, keeping at the same time the systematic uncertainties and background at low level. Assuming a 10\% signal acceptance and the SM decay rate, at least $10^{13}$ decays in the fiducial volume are required. This translates in the choice of a high intensity kaon beam: the same extraction line of the CERN SPS used for the NA48 experiment will deliver a $75 \mathrm{GeV} / \mathrm{c}$ charged kaon beam, fully adequate for NA62.

The NA62 guiding principles can be summarized as follows. The high rate environment demands high resolution timing. In addition, high resolution and fast tracking is needed to measure the momenta of the incoming kaon and of the daughter particles in order to exploit kinematic rejection by cutting on the missing mass at the decay. The rejection of decays with electrons and muons in the final state requires an efficient particle identification (PID) of all charged particle, namely the positive non-destructive identification of the parent kaon and PID of daughter particles (electrons, muons and pions) and also of muons not coming from the decay. To reject decay modes with $\pi^{0}$ and radiative photons in the final state, hermetic, high efficient $\gamma$ vetoing is required from 0 to $50 \mathrm{mrad}$. Furthermore redundancy of information will help to reach the goal. 


\subsection{Kinematic rejection}

The only measurable quantities for the decay under study are the momentum of the incoming kaon, $P_{K}$, the momentum of the outgoing pion, $P_{\pi}$, and the angle between the mother and the daughter particles, $\theta_{\pi K}$. The kinematic of the decay is schematically sketched in Fig. 4.

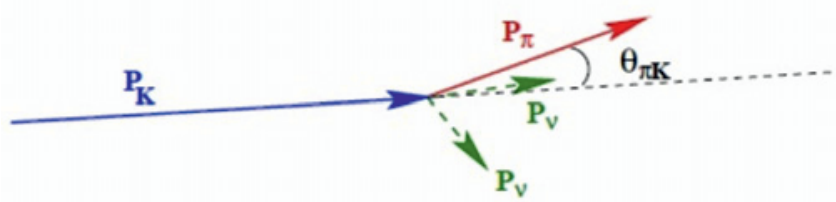

Figure 4. Kinematics of the $K^{+} \rightarrow \pi^{+} v \bar{v}$ decay.

It is convenient to use the squared missing mass variable, $m_{m i s s}^{2}$, defined under the hypothesis that the detected charged particle in the final state is a pion:

$$
m_{m i s s}^{2} \simeq m_{K}^{2}\left(1-\frac{\left|P_{\pi}\right|}{\left|P_{K}\right|}\right)+m_{\pi}^{2}\left(1-\frac{\left|P_{K}\right|}{\left|P_{\pi}\right|}\right)-\left|P_{K}\right|\left|P_{\pi}\right| \theta_{\pi K}^{2}
$$

In Fig. 5 the $m_{\text {miss }}^{2}$ for the signal and the kaon decays with the largest branching ratios are shown for $P_{K}=75 \mathrm{GeV} / \mathrm{c}$. If resolution effects are ignored, the $K^{+} \rightarrow \pi^{+} \pi^{0}$ decay is constrained to a line at $m_{m i s s}^{2}=m_{\pi^{0}}^{2}$; the $m_{m i s s}^{2}$ of the three-pion decays shows a lower bound. The $m_{m i s s}^{2}$ of $K^{+} \rightarrow \mu^{+} v$ does not appear as a line at $m_{\text {miss }}^{2}=0$ because it is wrongly evaluated, under the assumption that the track is a pion. In conclusion, about $92 \%$ of the background events can be separated from signal by kinematic cuts (see Fig. 5, left). For the remaining (see Fig. 5, right), the background events extend in the signal region and kinematic cannot help for the separation. The suppression, hence, has to rely on the photon veto systems and particle identification.
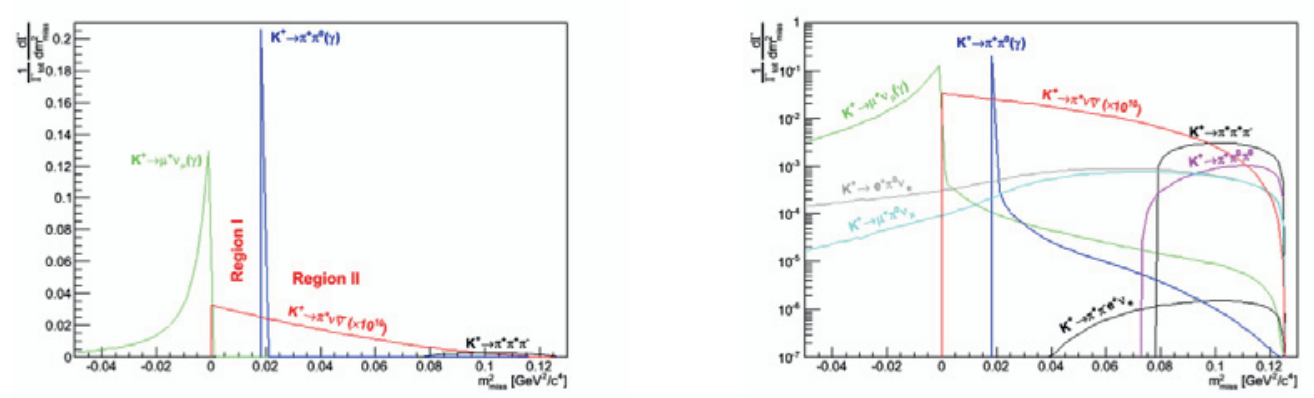

Figure 5. Squared missing mass for background decays that can be separated from the signal using kinematic cuts (left) and for decays where kinematics cannot be used (right). The backgrounds are normalized according to their branching ratios; the signal is multiplied by a factor $10^{10}$.

\subsection{Experiment and detectors}

The experimental setup is schematically depicted in Fig. 6. A $400 \mathrm{GeV} / \mathrm{c}$ primary proton beam extracted from the SPS impinges on a beryllium target to produce a secondary hadron beam at $75 \mathrm{GeV} / \mathrm{c}$ 
with a momentum bite of $\sim 1 \%$. Only $6 \%$ of the hadrons are charged kaons (unseparated beam). It is crucial to make a positive identification of the minority particles of interest, kaons, in the high rate beam environment before they decay. This is achieved by placing a differential Cherenkov counter (KTAG) in the incoming beam; the KTAG is insensitive to pions and protons with minimal accidental mis-tagging. In addition the KTAG detector is required to achieve a kaon identification efficiency of at least $95 \%$ and a time resolution of $100 \mathrm{ps}$, in conjunction with timing information from other detectors.

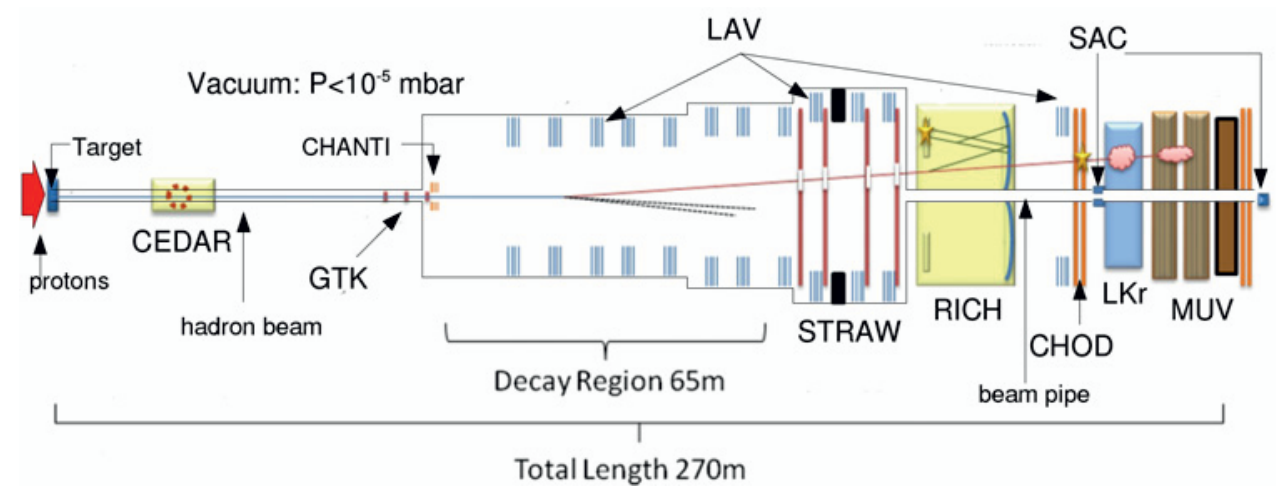

Figure 6. Detector layout

To fulfill the requirements needed for the kinematic rejection, keeping, at the same time, multiple scattering as low as possible, a beam tracker along the beam line, named Gigatracker (GTK), and a low mass spectrometer placed in vacuum downstream the decay region have been chosen. The GTK will be exposed to an instantaneous rate of $\sim 800 \mathrm{MHz}$. It consists of three silicon pixel stations, matching the beam size, placed before the decay volume, with 18000 pixels per station. Results from beam tests on prototypes [11] show a time resolution of $170 \mathrm{ps}$ for single hits from minimum ionizing particles, using $200 \mu \mathrm{m}$ thick silicon sensors, and a momentum resolution of $0.2 \%$, in agreement with the design requirements.

The direction and the momentum of secondary charged particles originating form the decay region will be measured by the straw tracker. The spectrometer consists of four chambers and a high aperture dipole magnet providing a vertical B-field of 0.36 T. Each chamber is equipped with 1792 straw tubes, which are positioned in four views [12].

The Photon Veto system is composed by several detectors, covering different angular regions. The Large Angle Veto (LAV) is made of lead glass blocks arranged in 12 annular rings around the decay volume. Photons from $K^{+} \rightarrow \pi^{+} \pi^{0}$ intercepted by the LAV may have energies from a few tens of $\mathrm{MeV}$ to several GeV's. In order to detect the $\pi^{0}$ 's with an inefficiency of $\leq 10^{-8}$, the maximum tolerable inefficiency in the LAV detectors for photons with energies as low as $200 \mathrm{MeV}$ is $10^{-4}$. In addition, the LAV detectors must have good time resolution $(\sim 1 \mathrm{~ns})$ and good energy resolution $(\sim 10 \%$ at 1 $\mathrm{GeV}$ ) and be compatible with operation in vacuum at $10^{-6}$ mbar. A detailed description of the LAV detector can be found in [13].

The intermediate angular region is covered by a quasi-homogenous calorimeter filled with liquid Krypton (LKr), inherited from NA48/2 (see [5]). 
Two small shashlik calorimeters are employed to veto photons at very small angles: the IRC covering the region around the inner radius of the $\mathrm{LKr}$ and the SAC situated behind the experimental cavern in the prolongation of the non-deviated axis covering the angular region down to zero degree.

A gas based RICH detector will contribute to the $\pi-\mu$ separation with a rejection factor better than $5 \times 10^{-3}$ in the momentum range $15-35 \mathrm{GeV} / \mathrm{c}$ and will also provide and event time measurement with a resolution better than $100 \mathrm{ps}$, in order to minimize wrong matching with the parent particle measured by an upstream detector. The RICH is a $18 \mathrm{~m}$ long segmented tube, $4 \mathrm{~m}$ in diameter, filled with $\mathrm{Ne}$ at atmospheric pressure and room temperature, equipped with a segmented mirror $(17 \mathrm{~m}$ focal length) at the downstream end and about 2000 Photomultipliers at the upstream end. Results from the beam tests are well within the expectations, showing a muon suppression factor of $0.7 \%$, very close to the requested $0.5 \%$ and a time resolution below 100 ps [14]. The detector is complemented with a Charged Hodoscope (CHOD), a Muon Detector (MUV) and a veto detector for charged particles at small angles (CHANTI).

\section{Conclusions and outlook}

Kaons offer unique opportunities to exploit precision physics, complementary to high-energy approach, to study the Standard Model and to search for New Physics. The new measurement of the $K^{ \pm} \rightarrow \pi^{ \pm} \gamma \gamma$ decay significantly improves the precision of the ChPT tests with this channel.

The construction of the NA62 detector is well advanced, installation will be completed by fall 2014. A first period of data taking is foreseen for the months of November and December 2014, when we expect to collect the first 10\% of the NA62 data set. In the years 2015 and 2016 the remainder of the data will be collected.

The analysis of the full data set will allow a measurement of the $\operatorname{BR}\left(K^{+} \rightarrow \pi^{+} v \bar{v}\right)$ with a precision of about $10 \%$. Any discrepancy with respect to the predicted value will be a clear signal of physics beyond the SM and will help to understand its flavour structure.

In addition, the high performances of the detectors can also be the building blocks for a further physics program.

\section{References}

[1] J. H. Christenson et al., Phys. Rev. Lett. 13, 138 (1964).

[2] G. Ecker, A. Pich, E. de Rafael Nucl. Phys. B 303, 665 (1988).

[3] G. D'Ambrosio and J. Portoles Nucl. Phys. B 386, 403 (1996).

[4] P. Kitching et al., Phys. Rev. Lett. 79, 4079 (1997).

[5] V. Fanti et al., Nucl. Instrum. Meth. A574, 433 (2007).

[6] J. Brod, M. Gorbahn and E. Stamou, Phys.Rev. D 83, 034030 (2011).

[7] G. Isidori, F. Mescia, P. Paradisi, C. Smith, S. Trine, HEP-PH 0604074. JHEP 0608, 064 (2006).

[8] M. Blanke et al., HEP-PH 0610298. JHEP 0701, 066 (2007).

[9] A. V. Artamonov et al., Phys. Rev. Lett. 101, 191802 (2008).

[10] G. Anelli et al., CERN-SPSC-2005-013, SPSC-P-326.

[11] M. Fiorini et al., Nucl. Instrum. Meth. A718, 270 (2013).

[12] H. Danielson et al., IEEE Nucl. Sci. Symp. 2010, 1914 (2010).

[13] P. Massarotti et al., PoS ICHEP 2012, 504 (2013).

[14] B. Angelucci et al., Nucl. Instrum. Meth. A621, 205 (2010). 